

\title{
INTERSECTIONS OF SETS AND FOURIER ANALYSIS
}

\author{
By \\ Suresh Eswarathasan, ${ }^{1}$ Alex Iosevich ${ }^{2}$ and Krystal Taylor ${ }^{3}$
}

\begin{abstract}
A classical theorem due to Mattila says that if $A, B \subset \mathbb{R}^{d}$ of Hausdorff dimension $s_{A}, s_{B}$ respectively with $s_{A}+s_{B} \geq d, s_{B}>(d+1) / 2$, and $\operatorname{dim}_{\mathcal{H}}(A \times B)=s_{A}+s_{B} \geq d$, then

$$
\operatorname{dim}_{\mathcal{H}}(A \cap(z+B)) \leq s_{A}+s_{B}-d
$$

for almost every $z \in \mathbb{R}^{d}$, in the sense of Lebesgue measure. In this paper, we replace the Hausdorff dimension on the left hand side of the first inequality above by the lower Minkowski dimension and replace the Lebesgue measure of the set of translates by a Hausdorff measure on a set of sufficiently large dimension. Interesting arithmetic issues arise in the consideration of sharpness examples.
\end{abstract}

\section{Introduction}

A series of results due to Mattila (see [7], [8], [9]; see also [10, Chapter 13]) give lower and upper bounds on the Hausdorff dimension of the intersection of subsets of euclidean space of a given Hausdorff dimension.

Theorem 1.1 ([10, p. 177]). Let $A$ and $B$ be Borel subsets of $\mathbb{R}^{d}, d \geq 2$, having positive Hausdorff dimension $s_{A}$ and $s_{B}$, respectively, such that $s_{A}+s_{B}>d$ and $s_{B}>(d+1) / 2$. Then, for almost every $g \in O(d)$ (the group of orthogonal $d \times d$ matrices),

$$
\mathcal{L}^{d}\left(\left\{z \in \mathbb{R}^{d}: \operatorname{dim}_{\mathcal{H}}(A \cap(z-g B)) \geq s_{A}+s_{B}-d\right\}\right)>0 .
$$

This means that for almost every rotation $g$, there exists a set of $z$ s of positive Lebesgue measure such that the Hausdorff dimension of $A \cap(z-g B)$ is at least

\footnotetext{
${ }^{1}$ The first listed author was supported by a CRM-ISM Postdoctoral Fellowship and McGill University during the first part of the writing of this article, with the second part written while as a resident at the Institute des Hautes Études Scientifiques.

${ }^{2}$ The work of the second listed author was partially supported by the NSF Grant DMS10-45404.

${ }^{3}$ The third listed author was supported by the Technion Technical Institute during the first part of the writing of this article, with second part written while at the Institute for Mathematics and its Applications.
} 
$s_{A}+s_{B}-d$. It is known that in a more restrictive setting, if either $A$ or $B$ is Salem, then the assumption that one of the dimensions is at least $(d+1) / 2$ is not necessary for $d \geq 2$ [10]. In general, the necessity of this condition is not known. Setting both $A$ and $B$ equal to the middle-1/3 Cantor set demonstrates that (1.1) fails for $d=1[10$, p. 179].

Theorem 1.2 ([10, p. 178]). Let $A$ and $B$ be Borel subsets of $\mathbb{R}^{d}, d \geq 2$, of Hausdorff dimension $s_{A}$ and $s_{B}$, respectively, satisfying

$$
\operatorname{dim}_{\mathcal{H}}(A \times B)=s_{A}+s_{B} \geq d .
$$

Then

$$
\operatorname{dim}_{\mathcal{H}}(A \cap(z-B)) \leq s_{A}+s_{B}-d
$$

for almost every $z \in \mathbb{R}^{d}$ in the sense of Lebesgue measure.

This tells us that if (1.2) holds, then the Hausdorff dimension of $A \cap(z-g B)$ is at most $s_{A}+s_{B}-d$ for $g \in O(d)$ and almost every $z \in \mathbb{R}^{d}$.

We produce an example in Remark 2.5 which illustrates that the assumption (1.2) in the previous theorem is a necessary condition.

A more general question, described in [10, pp. 139-145, 171-182] and the references contained therein, is the following.

Problem 1.3. Given $A, B$ subsets of $\mathbb{R}^{d}$ of suitable Hausdorff dimension, determine the Hausdorff dimension of $A \cap T(B)$, where and $T$ ranges contained a suitable set of transformations of $\mathbb{R}^{d}$.

Before giving a detailed description of the goals of this paper, we illustrate a simple motivating point by considering $A \cap(x-B)$, where $A, B \subset \mathbb{R}^{d}$. In order for the intersection to be non-empty, $x$ must be an element of the sum set $A+B$. If $A$ and $B$ are both sets of a given Hausdorff dimension less than $d$, the Hausdorff dimension of $A+B$ is also quite often less than $d$, and this naturally leads us to consider translates $x$ belonging to a set of a given Hausdorff dimension and to explore the thresholds for which the natural inequalities involving the dimension of $A \cap(x-B)$ hold. This simple point of view also indicates that the arithmetic properties of $A$ and $B$ play an important role.

An example of two sets $A$ and $B$ of Hausdorff dimension $s_{A}$ and $s_{B}$, respectively, such that the Hausdorff dimension of $A \cap(x-B)$ is "generically" $s_{A}+s_{B}-d$ is easily constructed by taking $A$ and $B$ to be smooth surfaces in $\mathbb{R}^{d}$. A simple example in the non-integer case is obtained by considering

$$
A=\left\{r \omega: \omega \in S^{d-1} ; r \in U\right\},
$$


where $U$ is an Ahlfors-David regular set of Hausdorff dimension $s_{U}$. It is not difficult to check that the Hausdorff dimension of $A$ is $d-1+s_{U}$. It is also straightforward to verify that the Hausdorff dimension of $A$ and every line that intersects $A$ is at most $s_{U}$.

We now describe the goals of this paper.

- Under structural assumptions on the sets $A$ and $B$, with $T_{z} B=B+z$, we prove that the set of translates $z$ for which the lower Minkowski dimension of $A \cap T_{z} B$ is larger than $\operatorname{dim}_{\mathcal{H}}(A)+\operatorname{dim}_{\mathcal{H}}(B)-d$ does not only have Lebesgue measure 0 but also small Hausdorff dimension. This is an analog of Theorem 1.2 above, in which finer information on the exceptional set of translates is obtained and the Hausdorff dimension on the left hand side is replaced with lower Minkowski dimension at the expense of additional assumptions on the set $B$.

- Without any additional assumptions on $A$ and $B$ beyond Ahlfors-David regularity, we replace the Hausdorff dimension in Theorem 1.2 with the lower Minkowski dimension.

- We obtain the same type of results for $T B=g B+z$, where $g \in O(d)$ and $z \in \mathbb{R}^{d}$. It turns out that for almost every $g \in O(d)$, the set of translates $z$ for which the lower Minkowski dimension of the set $A \cap T B$ is greater than $\operatorname{dim}_{\mathcal{H}}(A)+\operatorname{dim}_{\mathcal{H}}(B)-d$ has small Hausdorff dimension.

The main results of this paper are described in Section 2 below.

1.1 Notation. The following notation is used throughout the paper.

- $X \lesssim Y$ means that there exists $C>0$ independent of $X$ and $Y$ such that $X \leq C Y$.

- $X \lesssim Y$ with the controlling parameter $R$ means that given $\epsilon>0$, there exists $C_{\epsilon}>0$ such that $X \leq C_{\epsilon} R^{\epsilon} Y$.

- $B(x, \delta)$ is the ball of radius $\delta$ centered at $x \in \mathbb{R}^{d}$.

- For $A \subset \mathbb{R}^{d}, A^{\epsilon}$ is the open $\epsilon$-neighborhood of $A$.

- For non-empty $A \subset \mathbb{R}^{d}, s_{A}$ is the Hausdorff dimension of $A$, and $\mu_{A}$ is a probability measure on $A$. If $A$ is assumed to be Ahlfors-David regular, $\mu_{A}$ is the restriction of the $s_{A}$-dimensional Hausdorff measure to $A$.

- For a compactly supported measure $\mu$ on $\mathbb{R}^{d}$,

$$
I^{s}(\mu)=\iint|x-y|^{-s} d \mu(x) d \mu(y) \quad \text { (s-energy integral) }
$$

It follows from elementary properties of the Fourier transform that

$$
I^{s}(\mu)=\int|\widehat{\mu}(\xi)|^{2}|\xi|^{s-d} d \xi .
$$


If $0<\alpha<s$ and $\mu(B(x, \delta)) \leq C \delta^{s}$ for every $\delta>0$, then $I^{\alpha}(\mu) \lesssim 1$; see [2, p. 208] and [3, Section 6.2].

- For $A \subset \mathbb{R}^{d}, M(A)$ is the set of Radon measures $\mu$ with compact support contained in $A$ such that $0<\mu(A)<\infty$.

\section{Main results}

We work primarily with Ahlfors-David regular sets, defined as follows.

Definition 2.1. A Borel set $E \subset \mathbb{R}^{d}$ is Ahlfors-David regular if there exists $C>0$ such that for all $x \in E$,

$$
C^{-1} \delta^{s_{E}} \leq \mu(B(x, \delta)) \leq C \delta^{s_{E}}
$$

where $s_{E}$ is the Hausdorff dimension of $E$ and $\mu$ is the Hausdorff measure restricted to $E$.

2.1 Intersections of translated, rotated and dilated sets. We begin with the following variant of Theorem 1.2, where translation by $x \in \mathbb{R}^{d}$ is replaced by translation by $s(x)$ (a local diffeomorphism) and the Hausdorff dimension on the left hand side is replaced by the lower Minkowski dimension. This is done at the expense of assuming that the intersecting sets are Ahlfors-David regular.

Theorem 2.2. Suppose that $A$ and $B$ are compact, Ahlfors-David regular, Borel subsets of $\mathbb{R}^{d}, d \geq 2$, of Hausdorff dimension $s_{A}, s_{B}$, respectively, satisfying $s_{A}+s_{B} \geq d$. Denote by $\lambda(x)$ the lower Minkowski dimension of $A \cap(s(x)-B)$, where $s$ is a local $C^{\infty}$ diffeomorphism. Let $N(x, \epsilon)$ be the minimum number of open $\epsilon$-balls needed to cover $A \cap(s(x)-B)$. Then, for any smooth compactly supported function $\psi$ and every $\epsilon>0$, there exists a constant $C$, independent of $\epsilon$ and depending only on $\psi$ and s, such that

$$
\int N(x, \epsilon) \psi(x) d x \leq C\left(\epsilon^{-1}\right)^{s_{A}+s_{B}-d} .
$$

Thus

$$
\lambda(x) \leq s_{A}+s_{B}-d
$$

for almost every $x \in \mathbb{R}^{d}$.

Remark 2.3. In case $s(x) \equiv x$, Theorem 2.2 can be deduced from results in [4]. 
Remark 2.4. The assumption in the statement of Theorem 2.2 that $B$ is Ahlfors-David regular can be eliminated at the cost of using the Minkowski dimension of $B$ on the right hand side of the inequality instead of the Hausdorff dimension of $B$. The authors thank Brendan Murphy for this observation.

Remark 2.5. We address the sharpness of Theorem 2.2. The following example illustrates that the dimensional inequality (2.3) fails if the assumption that $A$ and $B$ are Ahlfors-David regular is dropped. In fact, we show that if $\operatorname{dim}(A \times B)>$ $\operatorname{dim}(A)+\operatorname{dim}(B)$ (which implies that neither $A$ nor $B$ is Ahlfors-David regular), then (2.3) fails even if the lower Minkowski dimension of the intersection set is replaced with the Hausdorff dimension of the intersection set.

Let $A=[0,2] \times Y$ and $B=X \times[0,2]$, where $X, Y \subset[0,1]$ are Borel sets satisfying

$$
\operatorname{dim}(X \times Y)>\operatorname{dim}(X)+\operatorname{dim}(Y) .
$$

Federer [5, pp. 191-194] constructed examples of such sets which are also Borel subsets of $[0,1]$. Observe that

$$
\begin{aligned}
A \cap B & =([0,2] \cap X) \times(Y \cap[0,2])=X \times Y, \\
A \cap(B+(u, v)) & =([0,2] \cap(X+u)) \times(Y \cap[v, 2+v])=(X+u) \times Y
\end{aligned}
$$

whenever $(u, v) \in[0,1] \times[-1,0]$. It follows by a result in [10] that since $X$ and $Y$ are Borel, $\operatorname{dim}(A)=1+\operatorname{dim}(Y)$ and $\operatorname{dim}(B)=1+\operatorname{dim}(X)$. Combining these observations, we conclude that

$$
\begin{aligned}
\operatorname{dim}(A \cap(B+(u, v))) & =\operatorname{dim}((X+u) \times Y)=\operatorname{dim}(X \times Y) \\
>\operatorname{dim}(X)+\operatorname{dim}(Y) & =\operatorname{dim}(A)+\operatorname{dim}(B)-2
\end{aligned}
$$

whenever $(u, v) \in[0,1] \times[-1,0]$. In other words, $(2.3)$ fails on a set of positive measure.

Combining Theorem 2.2 with Theorem 1.1, we deduce that the lower Minkowski dimension and the Hausdorff dimension of the intersection of an Ahlfors-David regular set with a rotated copy of a Borel set quite frequently coincide.

Corollary 2.6. Suppose that $A$ and $B$ are compact, Ahlfors-David regular, Borel subsets of $\mathbb{R}^{d}, d \geq 2$, of Hausdorff dimension $s_{A}, s_{B}$, respectively, which satisfy $s_{A}+s_{B} \geq d$. Also assume that $s_{B}>(d+1) / 2$. Then for almost every $g \in O(d)$,

$$
\mathcal{L}^{d}\left\{z \in \mathbb{R}^{d}: \operatorname{dim}_{\mathcal{H}}(A \cap(z-g B))=\underline{\operatorname{dim}}_{\mathcal{M}}(A \cap(z-g B))\right\}>0 .
$$


Remark 2.7. It is reasonable to conjecture that under the assumptions of Corollary 2.6, $A \cap(z-g B)$ is Ahlfors-David regular, but this does not follow from the equality of the Hausdorff and lower Minkowski dimensions. This can be seen by taking a Cantor construction and changing the dissection ratio at each stage. The second listed author thanks Pertti Mattila for pointing out this construction in the context of Ahlfors-David regularity.

Rotating $B$ before translating it, we discover that the exceptional set that was found in Theorem 2.2 above to have Lebesgue measure zero has small Hausdorff dimension.

Theorem 2.8. Suppose that $A$ and $B$ are compact, Ahlfors-David regular Borel subsets of $\mathbb{R}^{d}, d \geq 2$, of Hausdorf dimension $s_{A}, s_{B}$, respectively, which satisfy $s_{A}+s_{B} \geq d$. Let $\mu$ be a compactly supported probability measure such that $I^{\alpha}(\mu)<\infty$ for some $0<\alpha \leq d$ satisfying $\alpha+s_{A}>2(d-\kappa)$, where $\kappa=$ $\min \left\{(d-1) / 2, s_{B}\right\}$. Denote by $\lambda_{g}(x)$ the lower Minkowski dimension of $A \cap(x-g B)$, where $g \in O(d)$. Let $N(x, g, \epsilon)$ be the minimum number of open $\epsilon$-balls needed to cover $A \cap(x-g B)$. Then there exists $C>0$ such that for every $\epsilon>0$,

$$
\iint N(x, g, \epsilon) d \theta(g) d \mu(x) \leq C \sqrt{I^{\alpha}(\mu) I^{2(d-\kappa)-\alpha}\left(\mu_{A}\right)} \cdot\left(\epsilon^{-1}\right)^{s_{A}+s_{B}-d},
$$

where $d \theta(g)$ denotes normalized Haar measure on $O(d)$. Thus,

$$
\lambda_{g}(x) \leq s_{A}+s_{B}-d
$$

almost everywhere with respect to the probability measure $d \theta(g) d \mu(x)$. Furthermore,

$$
\operatorname{dim}_{\mathcal{H}}\left(\left\{x: \int \lambda_{g}(x) d \theta(g)>s_{A}+s_{B}-d\right\}\right) \leq d+1-s_{A} .
$$

We are also able to obtain a good upper bound on the Hausdorff dimension of the exceptional set if we put additional structural assumptions on one of the sets being intersected.

Definition 2.9. A compact Borel set $B \subset \mathbb{R}^{d}$ of Hausdorff dimension $s_{B}$ satisfies the hyperplane size condition of order $h$ for some $0<h<s_{B}$ if there exists a Borel measure $\mu_{B}$ supported in $B$ such that $\mu_{B}\left(H_{\omega}^{\delta}\right) \leq C \delta^{s_{B}-h}$, where $H_{\omega}=\left\{x \in \mathbb{R}^{d}: x \cdot \omega=0\right\}$.

Remark 2.10. If $\mu_{B}$ is a Frostman measure (see [10, pp. 112-114]), then the hyperplane size condition with $h=d-1$ always holds. This is because the intersection of $B$ with a hyperplane can be decomposed into approximately $\delta^{-(d-1)}$ 
$\delta$-cells and the measure of each cell is at most $C \delta^{s_{B}}$ by the Frostman property. One should think of $h$ as an upper bound on the dimension of the intersection of $B$ with a $(d-1)$-dimensional hyperplane.

Theorem 2.11. Suppose that $A$ and $B$ are compact, Ahlfors-David regular Borel subsets of $\mathbb{R}^{d}, d \geq 2$, of Hausdorff dimension $s_{A}, s_{B}$, respectively, which satisfy $s_{A}+s_{B} \geq d$. Let $\mu$ be a compactly supported probability measure satisfying $I^{\alpha}(\mu)<\infty$. Furthermore, suppose that $B$ satisfies the hyperplane size condition of order $h<s_{B}$, and $\left(\alpha+s_{A}\right) / 2>d-\left(s_{B}-h\right)$. Denote by $\lambda_{t}(x)$ the lower Minkowski dimension of $A \cap(x-t B)$. Let $N(x, t, \epsilon)$ be the minimum number of open $\epsilon$-balls needed to cover $A \cap(x-t B)$. Then

$$
\iint_{1}^{2} N(x, t, \epsilon) d t d \mu(x) \leq C\left(\epsilon^{-1}\right)^{s_{A}+s_{B}-d} .
$$

Thus

$$
\lambda_{t}(x) \leq s_{A}+s_{B}-d
$$

almost everywhere with respect to the probability measure $d t d \mu(x)$ and $t \in[1,2]$. Finally, for almost every $t \in[1,2]$,

$$
\operatorname{dim}_{\mathcal{H}}\left(\left\{x: \int_{1}^{2} \lambda_{t}(x) d t>s_{A}+s_{B}-d\right\}\right) \leq 2\left(d-\left(s_{B}-h\right)\right)-s_{A}
$$

\section{Proof of Theorem 2.2}

Let $\mu_{A}$ and $\mu_{B}$ be the restrictions of the $s_{A^{-}}$and $s_{B}$-dimensional Hausdorff measures to $A$ and $B$, respectively, normalized so that $\int d \mu_{A}=\int d \mu_{B}=1$. Take $\psi \in C_{0}^{\infty}\left(\mathbb{R}^{d}\right)$. We prove (2.2) by obtaining upper and lower bounds on

$$
\int \mu_{A}\left(\{A \cap(s(x)-B)\}^{\epsilon}\right) \psi(x) d x
$$

To obtain a lower bound, we utilize the following lemma.

Lemma 3.1. Let $A, B$, and $s$ be as in Theorem 2.2. For fixed $\epsilon>0$, set $g(x)=N(x, \epsilon)$, where $N(x, \epsilon)$ is equal to the minimum number of open $\epsilon$-balls needed to cover $A \cap(s(x)+B)$. Then $g(x)$ is an upper semi-continuous function on $\mathbb{R}^{d}$. Hence $N(x, \epsilon)$ is measurable on $\mathbb{R}^{d}$.

The proof of Lemma 3.1 is given in the Appendix.

We multiply each side of

$$
N(x, \epsilon) \epsilon^{s_{A}} \lesssim \mu_{A}\left(\{A \cap(s(x)-B)\}^{\epsilon}\right)
$$


by $\psi(x)$, integrate with respect to $x$ (which is allowed by Lemma 3.1), and use the Ahlfors-David regularity of $A$ to conclude that (3.1) is bounded below by

$$
C \epsilon^{s_{A}} \int N(x, \epsilon) \psi(x) d x,
$$

where $C$ is a constant depending only on the dimension.

To obtain an upper bound on (3.1), we observe that

$$
(A \cap(s(x)-B))^{\epsilon} \subset\left\{y \in A^{\epsilon}: s(x)-y \in B^{\epsilon}\right\} ;
$$

therefore, (3.1) is bounded above by

$$
\int \mu_{A}\left(\left\{y \in A^{\epsilon}: s(x)-y \in B^{\epsilon}\right\}\right) \psi(x) d x .
$$

Denote by $J_{s}$ the Jacobian of the change of variables $x \rightarrow s(x)$, and notice that

$$
\int \mu_{A}\left\{y \in A^{\epsilon}: s(x)-y \in B^{\epsilon}\right\} \psi(x) d x \approx \iint \chi_{B^{\epsilon}}(s(x)-y) d \mu_{A}(y) \psi(x) d x,
$$

where $\chi_{B^{\epsilon}}$ is some cut-off function supported in a small neighborhood of $B^{\epsilon}$. Using properties of the Fourier transform, we obtain the bound

$\left|\iint \chi_{B^{\epsilon}}(s(x)-y) d \mu_{A}(y) \psi(x) d x\right| \leq \int\left|\widehat{\chi_{B^{\epsilon}}}(\xi)\right| \cdot\left|\widehat{\mu}_{A}(\xi)\right| \cdot \mid\left(J_{s^{-1}} \cdot\left(\psi \circ s^{-1}\right) \hat{)}(\xi) \mid d \xi\right.$.

Denote by $\mathcal{L}^{d}\left(B^{\epsilon}\right)$ the $d$-dimensional Lebesgue measure of the set $B^{\epsilon}$. Since $\left\|\widehat{\mu}_{A}\right\|_{\infty} \leq \int d \mu_{A}(x)=1, \psi \circ s^{-1}$ is a smooth and compactly supported function and $\left\|\widehat{\chi_{B^{\epsilon}}}\right\|_{\infty} \leq\left\|\chi_{B^{\epsilon}}\right\|_{1}$, we further bound the expression above by

$$
C \mathcal{L}^{d}\left(B^{\epsilon}\right)
$$

where $C$ is independent of $\epsilon$.

Let $N(B, \epsilon)$ be the minimum number of open $\epsilon$-balls needed to cover $B$. Recall (e.g., by the Ahlfors-David regularity of $B$ ) that the Hausdorff dimension of $B$ equals the Minkowski dimension of $B$. Using the definition of the Minkowski dimension, we see that $\mathcal{L}^{d}\left(B^{\epsilon}\right) \sim \epsilon^{d} N(B, \epsilon) \sim \epsilon^{d-s_{B}}$. Now the expression in (3.3) is $\sim c \epsilon^{d-s_{B}}$.

Comparing our upper and lower bounds, we see that for any smooth compactly supported function $\psi$, any smooth diffeomorphism $s$ of $\mathbb{R}^{d}$, and every $\epsilon>0$,

$$
\int N(x, \epsilon) \psi(x) d x \lesssim \epsilon^{-\left(s_{A}+s_{B}-d\right)} .
$$

This is precisely (2.2).

The proof of (2.3) is presented in the Appendix. 


\section{Proof of Theorem 2.8}

Denote by $\mu_{A}$ and $\mu_{B}$ the restrictions of the $s_{A^{-}}$and $s_{B}$-dimensional Hausdorff measures to $A$ and $B$, respectively, normalized so that $\int d \mu_{A}=\int d \mu_{B}=1$. We prove (2.6) by obtaining upper and lower bounds on

$$
\iint \mu_{A}\left(\{A \cap(x-g B)\}^{\epsilon}\right) d \mu(x) d \theta(g) .
$$

Let $N(x, g, \epsilon)$ be the minimum number of open $\epsilon$-balls needed to cover $A \cap(x-g B)$. It follows from the properties of $\mu_{A}$, which are a consequence of the Ahlfors-David regularity of $A$, that

$$
N(x, g, \epsilon) \epsilon^{s_{A}} \lesssim \mu_{A}\left(\{A \cap(x-g B)\}^{\epsilon}\right) .
$$

Integrating each side of this with respect to the measures $d \mu$ and $d \theta$, we conclude that (4.1) is bounded below by

$$
\iint N(x, g, \epsilon) \epsilon^{s_{A}} d \mu(x) d \theta(g) .
$$

The measurability of $N(x, g, \epsilon)$ follows from arguments similar to those used in the proof of Lemma 3.1, applied to local coordinates on $O(d)$.

Next, observe that $\{A \cap(x-g B)\}^{\epsilon} \subset\left\{y \in A^{\epsilon}: x-y \in g B^{\epsilon}\right\}$; and so (4.1) is bounded above by

$$
\iint \mu_{A}\left\{y \in A^{\epsilon}: x-y \in g B^{\epsilon}\right\} d \mu(x) d \theta(g),
$$

which is comparable to

$$
\begin{aligned}
& \iiint \chi_{B^{\epsilon}}(g(x-y)) d \mu(x) d \mu_{A}(y) d \theta(g) \\
&=\int\left(\int \widehat{\chi_{B^{\epsilon}}}(g \xi) d \theta(g)\right) \widehat{\widehat{\mu}(\xi)} \widehat{\mu}_{A}(\xi) d \xi .
\end{aligned}
$$

Lemma 4.1. With the notation above,

$$
\left|\int \widehat{\chi_{B^{\epsilon}}}(g \xi) d \theta(g)\right| \lesssim \epsilon^{d-s_{B}}(1+|\xi|)^{-\kappa},
$$

where $\kappa=\min \left\{(d-1) / 2, s_{B}\right\}$.

We postpone the proof of Lemma 4.1 to the end of this section.

The lemma allows us to bound (4.3) by

$$
\epsilon^{d-s_{B}} \int|\xi|^{-\kappa}|\widehat{\mu}(\xi)| \cdot\left|\widehat{\mu}_{A}(\xi)\right| d \xi .
$$


Now write $-\kappa=\frac{(\alpha-d)}{2}+\left(-\kappa-\frac{(\alpha-d)}{2}\right)$. By the Cauchy-Schwarz inequality, (4.4) is bounded by

$$
\epsilon^{d-s_{B}} \sqrt{I^{\alpha}(\mu) I^{2(d-\kappa)-\alpha}\left(\mu_{A}\right)} \lesssim \epsilon^{d-s_{B}}
$$

Indeed, $I^{\alpha}(\mu)$ is finite by assumption, and $I^{2(d-\kappa)-\alpha}\left(\mu_{A}\right) \lesssim 1$ follows from the hypothesis that $s_{A}+\alpha>2(d-\kappa)$.

Combining the upper and lower bounds (4.5) and (4.2), respectively, for (4.1), we obtain

$$
\epsilon^{s_{A}} \iint N(x, \epsilon) d \mu(x) d \theta(g) \lesssim \sqrt{I^{\alpha}(\mu) I^{2(d-\kappa)-\alpha}\left(\mu_{A}\right)} \epsilon^{d-s_{B}},
$$

which is precisely (2.6)

Next, observe that (2.7) follows from (2.6). The proof is almost identical to that of (2.3) The only changes necessary are to replace $d x$ with $d \theta(g) d \mu(x)$ and the words "positive Lebesgue measure" with "positive measure with respect to $d \theta(g) d \mu(x) "$.

To prove (2.8), assume by way of contradiction that the exceptional set $E=\left\{x: \lambda_{g}(x)>s_{A}+s_{B}-d\right\}$ has dimension larger than $d+1-s_{A}$. Assume also that $d+1-s_{A}<d$; otherwise, the claim holds trivially. Choose $\alpha$ such that

$$
d+1-s_{A}<\alpha<\operatorname{dim}_{\mathcal{H}}\left\{x: \int \lambda_{g}(x) d \theta(g)>s_{A}+s_{B}-d\right\} .
$$

By Frostman's Lemma, [10, pp. 112-114], there exists a compactly supported probability measure $\mu$ with support contained in $E$ satisfying $I^{\alpha}(\mu)<\infty$. Observing that (2.7) holds for $\mu$, we arrive at a contradiction. This concludes the proof of Theorem 2.8, given Lemma 4.1.

\subsection{Proof of Lemma 4.1. We have}

$$
\int \widehat{\chi_{B^{\epsilon}}}(g \xi) d \theta(g)=\iint e^{-2 \pi i g^{-1} x \cdot \xi} d \theta(g) \chi_{B^{\epsilon}}(x) d x=c \int \widehat{\sigma}(|x| \xi) \chi_{B^{\epsilon}}(x) d x,
$$

where $\sigma$ is Lebesgue measure on the unit sphere $S^{d-1}$ in $\mathbb{R}^{d}$ and $c$ is a constant depending only on $B$. Using the well-known estimate of the Fourier transform of the sphere, $|\widehat{\sigma}(\xi)| \lesssim(1+|\xi|)^{-(d-1) / 2}$, we bound the modulus of (4.6) by a constant times

$$
\int(1+(|x||\xi|))^{-(d-1) / 2} \chi_{B^{\epsilon}}(x) d x .
$$

We consider the two cases $|\xi| \leq 1$ and $|\xi|>1$ separately. 
In case $|\xi| \leq 1,(4.7)$ is bounded by $\int \chi_{B^{\epsilon}}(x) d x$. Now we approximate the $d$-dimensional Lebesgue measure of the set $B^{\epsilon}$ by the minimal number of $\epsilon$-balls needed to cover $B$ times the size of such a ball, $\epsilon^{d}$. Since $B$ is Ahlfors-David regular, the Hausdorff dimension of $B$ equals the lower Minkowski dimension of $B$; and so the $d$-dimensional Lebesgue measure of $B^{\epsilon}$ is approximately $\epsilon^{d-s_{B}}$. This proves the lemma when $|\xi| \leq 1$.

In case $|\xi|>1$, we break up the integral in (4.7) into three regions:

$$
\left\{x:|x|<\frac{1}{|\xi|}\right\}, \quad\left\{x: \frac{1}{|\xi|}<|x|<1\right\}, \quad \text { and }\{x: 1<|x|\}
$$

and bound (4.7) from above by

$$
\begin{aligned}
\int_{\left\{x:|x|<\frac{1}{|\xi|}\right\}} \chi_{B^{\epsilon}}(x) d x & +\int_{\left\{x: \frac{1}{|\xi|}<|x|<1\right\}}(|x||\xi|)^{-(d-1) / 2} \chi_{B^{\epsilon}}(x) d x \\
& +\int_{\{x: 1<|x|\}}(|x||\xi|)^{-(d-1) / 2} \chi_{B^{\epsilon}}(x) d x \\
=: I & +I I+I I I .
\end{aligned}
$$

In each region, we utilize the following elementary estimates.

Proposition 4.2. Let $\rho: \mathbb{R}^{d} \rightarrow \mathbb{R}$ be a non-negative, smooth, compactly supported function satisfying $\rho(x) \geq 1$ for $x \in B(0,2)$. For $\epsilon>0$, let $\rho_{\epsilon}(x)=$ $\rho(x / \epsilon) / \epsilon^{d}$. Let $B$ be an Alhfors-David regular set. Then, for $\delta>0$,

$$
\begin{gathered}
\chi_{B^{\epsilon}}(x) \lesssim \epsilon^{d-s_{B}} \mu_{B} * \rho_{\epsilon}(x), \\
\int_{\{x:|x|<\delta\}} \mu_{B} * \rho_{\epsilon}(x) d x \lesssim \delta^{s_{B}}, \\
\int \mu_{B} * \rho_{\epsilon}(x) d x \lesssim 1 .
\end{gathered}
$$

These estimates are standard and are left as an exercise for the reader.

Using Proposition 4.2, we obtain

$$
\begin{aligned}
I= & \int_{\left\{x:|x|<\frac{1}{|\xi|}\right\}} \chi_{B^{\epsilon}}(x) d x \lesssim \epsilon^{d-s_{B}} \int_{\left\{x:|x|<\frac{1}{|\xi|}\right\}} \mu_{B} * \rho_{\epsilon}(x) d x \lesssim \epsilon^{d-s_{B}}|\xi|^{-s_{B}} \\
I I= & \int_{\left\{x: \frac{1}{|\xi|}<|x|<1\right\}}(|x||\xi|)^{-\frac{(d-1)}{2}} \chi_{B^{\epsilon}}(x) d x \lesssim \epsilon^{d-s_{B}} \int_{\left\{x: \frac{1}{|\xi|}<|x|<1\right\}}(|x||\xi|)^{-\frac{(d-1)}{2}} \mu_{B} * \rho_{\epsilon}(x) d x \\
& \sim \epsilon^{d-s_{B}}|\xi|^{-\frac{(d-1)}{2}} \sum_{j=0}^{\log _{2}\left(\frac{1}{|\xi|}\right)-1} \int_{\left\{x: 2^{-(j+1)}<|x|<2^{-j}\right\}}|x|^{-\frac{(d-1)}{2}} \mu_{B} * \rho_{\epsilon}(x) d x \\
& \lesssim \epsilon^{d-s_{B}}|\xi|^{-\frac{(d-1)}{2}} \sum_{j=0}^{\log _{2}\left(\frac{1}{|\xi|}\right)-1} 2^{j\left(\frac{(d-1)}{2}-s_{B}\right)} \lesssim \epsilon^{d-s_{B}}|\xi|^{-\kappa},
\end{aligned}
$$


where $\kappa=\min \left\{\frac{d-1}{2}, s_{B}\right\}$; and

$$
\begin{aligned}
I I I & =\int_{\{x: 1<|x|\}}(|x||\xi|)^{-\frac{(d-1)}{2}} \chi_{B^{\epsilon}}(x) d x \lesssim \epsilon^{d-s_{B}}|\xi|^{-\frac{(d-1)}{2}} \int_{\{x:|x|>1\}}|x|^{-\frac{(d-1)}{2}} \mu_{B} * \rho_{\epsilon}(x) d x \\
& \lesssim \epsilon^{d-s_{B}}|\xi|^{-\frac{(d-1)}{2}} \int \mu_{B} * \rho_{\epsilon}(x) d x \lesssim \epsilon^{d-s_{B}}|\xi|^{-\frac{(d-1)}{2}} .
\end{aligned}
$$

This concludes the proof of Lemma 4.1.

\section{Proof of Theorem 2.11}

We prove (2.9) by obtaining upper and lower bounds on

$$
\iint_{1}^{2} \mu_{A}\left(\{A \cap(x-t B)\}^{\epsilon}\right) d t d \mu(x) .
$$

Let $N(x, t, \epsilon)$ be the minimum number of open $\epsilon$-balls needed to cover $A \cap(x-t B)$. By the Ahlfors-David regularity of $A$,

$$
N(t, x, \epsilon) \epsilon^{s_{A}} \lesssim \mu_{A}\left(\{A \cap(x-t B)\}^{\epsilon}\right) .
$$

Integrating each side of this with respect to the measure $d t d \mu(x), t \in(1,2)$, we conclude that (5.1) is bounded below by

$$
\epsilon^{s_{A}} \iint_{1}^{2} N(x, t, \epsilon) d t d \mu(x) .
$$

Next observe that $\{A \cap(x-t B)\}^{\epsilon} \subset A^{\epsilon} \cap\left(x-t B^{\epsilon}\right)$. Hence, (5.1) is bounded above by

$$
\iint_{1}^{2} \mu_{A}\left(A^{\epsilon} \cap\left(x-t B^{\epsilon}\right)\right) d t d \mu(x) .
$$

Let $\rho: \mathbb{R}^{d} \rightarrow \mathbb{R}$ be a smooth-bump function satisfying $\rho(x) \geq 1$ for $x \in$ $B(0,1 / 2)$ and vanishing outside of $B(0,1)$. Then

$$
\mu_{A}\left(A^{\epsilon} \cap\left(x-t B^{\epsilon}\right)\right) \lesssim \epsilon^{d-s_{B}} \int \mu_{B} * \rho_{4 \epsilon}\left(\frac{x-y}{t}\right) d \mu_{A}(y) .
$$

The measurability of $N(x, t, \epsilon)$ follows once again from arguments similar to those of Lemma 3.1. Integrating in $x$ with respect to the measure $\mu$ and in $t \in[1,2]$, we bound (5.1) from above by

$$
\epsilon^{-\left(s_{B}-d\right)} \iiint \mu_{B} * \rho_{4 \epsilon}\left(\frac{x-y}{t}\right) \psi(t) d \mu_{A}(y) d t d \mu(x),
$$

where $\psi$ is a translated smooth bump function equal to one on [1,2] and vanishing outside of $[0.5,2.5]$. 
Lemma 5.1. With the notation above,

$$
\iiint \mu_{B} * \rho_{\epsilon}\left(\frac{x-y}{t}\right) \psi(t) d \mu_{A}(y) d t d \mu(x) \lesssim 1,
$$

whenever $\left(s_{A}+\alpha\right) / 2>d-\left(s_{B}-h\right)$.

Assuming Lemma 5.1 for the moment, we have proved that (5.1) is bounded above by a constant times $(1 / \epsilon)^{s_{B}-d}$ and below by a constant times (5.3), i.e.,

$$
\epsilon^{s_{A}} \iint_{1}^{2} N(x, t, \epsilon) d t d \mu(x) \lesssim \epsilon^{-\left(s_{B}-d\right)},
$$

which proves (2.9).

Once again, we observe that (2.10) follows from (2.9); the proof is almost identical to that of (2.3)). The only changes that need to be made are to replace $d x$ with $d t d \mu(x)$ for $t \in[1,2]$, the smooth cut off function $\psi$ with the constant function 1, and the words "positive Lebesgue measure" with "positive measure with respect to $d t d \mu(x)$ "'.

To prove (2.11), we follow same method as in the previous proofs. Once again, we assume that the exceptional set $E=\left\{x: \lambda_{t}(x)>s_{A}+s_{B}-d\right\}$ has dimension larger than $2 d-2 s_{B}+2 h-s_{A}$ and arrive at a contradiction. We also assume that $2 d-2 s_{B}+2 h-s_{A}<d$; otherwise the claim holds trivially. Let

$$
2 d-2 s_{B}+2 h-s_{A}<\alpha<\operatorname{dim}_{\mathcal{H}}\left(\left\{x: \lambda_{t}(x)>s_{A}+s_{B}-d\right\}\right) .
$$

By Frostman's Lemma, there exists a compactly supported probability measure $\mu$ with support contained in $E$ satisfying $I^{\alpha}(\mu)<\infty$. We use (2.10) to arrive at a contradiction.

To finish the proof of the theorem, it remains to prove Lemma 5.1. Using elementary properties of the Fourier transform, we rewrite the left-hand side of (5.7) as

$$
\iint\left(\mu_{B} * \rho_{\epsilon}\right) \widehat{(t \xi)} \widehat{\mu_{A}}(\xi) \overline{\widehat{\mu}(\xi)} t^{d} \psi(t) d t d \xi
$$

The modulus of this expression is bounded above by a constant times

$$
\int\left|\widehat{\mu_{A}}(\xi)\right||\widehat{\mu}(\xi)|\left|\int\left(\mu_{B} * \rho_{\epsilon}\right) \hat{(}(\xi) t^{d} \psi(t) d t\right| d \xi .
$$

The following estimate is a key point towards establishing our lemma.

Proposition 5.2. With the notation above, for all $s_{B}-h>\eta>0$, there exists $c_{\eta}$, independent of $\epsilon$, such that

$$
\mid \int\left(\left.\mu_{B} * \rho_{\epsilon} \hat{)}(t \xi) t^{d} \psi(t) d t\left|\lesssim c_{\eta}\right| \xi\right|^{-\left(s_{B}-h\right)+\eta} .\right.
$$


We postpone the proof of Proposition 5.2 to the end of this section.

Using Proposition 5.2, we can now bound (5.9) above by a constant times

$$
\int\left|\widehat{\mu_{A}}(\xi)\right| \cdot|\widehat{\mu}(\xi)| \cdot|\xi|^{-\left(s_{B}-h\right)+\eta} d \xi
$$

Write $-\left(s_{B}-h\right)=\frac{\alpha-d}{2}-\left(s_{B}-h\right)-\frac{\alpha-d}{2}$. It follows by the Cauchy-Schwarz inequality that (5.11) is bounded above by

$$
\sqrt{I^{\alpha}(\mu) I^{2\left(d-\left(s_{B}-h\right)+\eta\right)-\alpha}\left(\mu_{A}\right)} .
$$

By assumption, $I^{\alpha}(\mu) \lesssim 1$. Observing that $s_{A}+\alpha>2\left(d-\left(s_{B}-h\right)\right)$ implies that $I^{2\left(d-\left(s_{B}-h\right)+\eta\right)-\alpha}\left(\mu_{A}\right) \lesssim 1$ for sufficiently small $\eta>0$, we conclude that (5.12) is bounded by a positive constant which does not depend on $\epsilon$ whenever $\left(s_{A}+\alpha\right) / 2>$ $d-\left(s_{B}-h\right)$. This completes the proof of Lemma 5.1, up to the proof of Proposition 5.2 .

\subsection{Proof of Proposition 5.2 Consider}

$$
\int\left(\mu_{B} * \rho_{\epsilon}\right) \hat{(t \xi) t} t^{d} \psi(t) d t
$$

Motivated by the presence of $\widehat{\rho_{\epsilon}}(t \xi)$, we consider the cases $|\xi|<1 / \epsilon$ and $|\xi|>1 / \epsilon$ separately.

In case $|\xi|<1 / \epsilon$, set $\tilde{\psi}=t^{d} \psi$ and $\mu_{B}^{\epsilon}=\mu_{B} * \rho_{\epsilon}$. Using the definition of the Fourier transform, we rewrite (5.13) as

$$
\int\left(\mu_{B}^{\epsilon}\right) \hat{(t \xi)} \tilde{\psi}(t) d t=\int \widehat{\tilde{\psi}}(x \cdot \xi) \mu_{B}^{\epsilon}(x) d x .
$$

Next, we use the rapid decay of $\tilde{\psi}$ to bound the modulus of this expression by a constant $C_{N}$ times

$$
\int(1+|x \cdot \xi|)^{-N} \mu_{B}^{\epsilon}(x) d x
$$

for $N>1$. We break this integral into integrals over the regions $\{x:|x \cdot \xi|<1\}$ and $\{x:|x \cdot \xi|>1\}$, i.e., we estimate (5.15) above by

$$
\int_{\{x:|x \cdot \xi|<1\}} \mu_{B}^{\epsilon}(x) d x+\int_{\{x:|x \cdot \xi|>1\}}|x \cdot \xi|^{-N} \mu_{B}^{\epsilon}(x) d x=I+I I .
$$

Considering (5.15) restricted to the first region, we write out the integrand explicitly as

$$
I=\int_{\{x:|x \cdot \xi|<1\}} \mu_{B}^{\epsilon}(x) d x=\frac{1}{\epsilon^{d}} \iint_{\{x:|x \cdot \xi| \leq 1\}} \rho\left(\frac{x-y}{\epsilon}\right) d x d \mu_{B}(y) .
$$


Fix $y$, recall that $\rho$ is supported in $B(0,1)$, and break the integral in $x$ into integrals over the regions $\{x:|x-y|<\epsilon\}$ and $\{x: 1>|x-y|>\epsilon\}$. Then, for $M>1$, we have the further decomposition

$$
\begin{aligned}
I \lesssim & \frac{1}{\epsilon^{d}} \iint_{\{x:|x \cdot \xi| \leq 1\} \cap\{x:|x-y|<\epsilon\}} d x d \mu_{B}(y) \\
& +\iint_{\{x:|x \cdot \xi| \leq 1\} \cap\{x: 1>|x-y|>\epsilon\}}\left|\frac{x-y}{\epsilon}\right|^{-M} d x d \mu_{B}(y) \\
& =I_{a}+I_{b} .
\end{aligned}
$$

Observe that

$$
\begin{aligned}
I_{a} & =\frac{1}{\epsilon^{d}} \iint_{\{x:|x \cdot \xi| \leq 1\} \cap\{x:|x-y|<\epsilon\}} d x d \mu_{B}(y) \lesssim \mu_{B}(\{y:|y \cdot \xi| \leq 1+\epsilon|\xi|\}) \\
& \lesssim \mu_{B}\left(\left\{y:\left|y \cdot \frac{\xi}{|\xi|}\right| \leq \frac{2}{|\xi|}\right\}\right),
\end{aligned}
$$

where the last line follows since we assumed that $|\xi|<1 / \epsilon$. Applying the hyperplane size condition of order $h$ on the set $B$, we conclude that $I_{a} \lesssim|\xi|^{-\left(s_{B}-h\right)}$.

Similarly, we have

$$
\begin{aligned}
I_{b} & =\frac{1}{\epsilon^{d}} \iint_{\{x:|x \cdot \xi| \leq 1\} \cap\{x: 1>|x-y|>\epsilon\}}\left|\frac{x-y}{\epsilon}\right|^{-M} d x d \mu_{B}(y) \\
& \approx \epsilon^{M-d} \sum_{j=0}^{\log _{2}(1 / \epsilon)-1} \iint_{\{x:|x \cdot \xi| \leq 1\} \cap\left\{x: 2^{-(j+1)}<|x-y|<2^{-j}\right\}} 2^{j M} d x d \mu_{B}(y) \\
& \lesssim \epsilon^{M-d} \sum_{j=0}^{\log _{2}(1 / \epsilon)-1} 2^{j M} 2^{-j d} \mu_{B}\left(\left\{y:\left|y \cdot \frac{\xi}{|\xi|}\right| \leq 2^{-j}+\epsilon\right\}\right) .
\end{aligned}
$$

Once again applying the hyperplane size condition of order $h$ on the set $B$, we may bound this quantity by

$$
\epsilon^{M-d} \sum_{j=0}^{\log _{2}(1 / \epsilon)-1} 2^{j\left(M-d-\left(s_{B}-h\right)\right)} \approx \epsilon^{s_{B}-h},
$$

for $M$ sufficiently large. Reminding ourselves that $|\xi|<1 / \epsilon$, we conclude that $I_{b} \lesssim|\xi|^{-\left(s_{B}-h\right)}$.

Moving onto the second region in the case $|\xi|<1 / \epsilon$, we consider

$$
I I=\int_{\{x:|x \cdot \xi|>1\}}|x \cdot \xi|^{-N} \mu_{B}^{\epsilon}(x) d x .
$$


Breaking the integral further into integrals over the regions $\left\{x: 2^{k}<|x \cdot \xi|<2^{k+1}\right\}$, for $k \in \mathbb{N}$ and using estimates nearly identical to those used for bounding $I$, we can show that $I I \lesssim|\xi|^{-\left(s_{B}-h\right)}$. We omit the details.

It remains to consider the case $|\xi|>1 / \epsilon$. We consider separately the two subcases $|\xi|>(1 / \epsilon)^{1+1 / c}$ and $(1 / \epsilon)^{1+1 / c}>|\xi|>1 / \epsilon$, where a positive lower bound on $c$ will be determined and shown to be independent of $\epsilon$. We obtain two estimates on the modulus of (5.13) which are used in both cases. Revisiting the estimates for $I_{a}, I_{b}$, and $I_{c}$ with the assumption that $|\xi|>1 / \epsilon$, we see that the modulus of (5.13) is bounded by another dimensional constant times $\epsilon^{s_{B}-h}$. On the other hand, using the rapid decay of $\widehat{\rho}$, we bound the modulus of the expression in (5.13) by a dimensional constant times $C_{N} \cdot(\epsilon|\xi|)^{-N}$ for $N \geq 1$. Indeed,

$$
\int\left|\widehat{\mu_{B}}(t \xi)\right||\widehat{\rho}(\epsilon t \xi)| t^{d} \psi(t) d t \lesssim C_{N} \int(1+\epsilon t|\xi|)^{-N} t^{d} \psi(t) d t
$$

for $N \geq 1$. Because we are assuming that $|\xi|>1 / \epsilon$ and since $\psi$ vanishes outside of $[0.5,2.5]$, we see that $\epsilon t|\xi| \geq \epsilon|\xi| / 2$ on $[1,2]$, and so we have the upper bound on (5.13) over the indicated region

$$
C_{N}(\epsilon|\xi|)^{-N} \int t^{d-N} \psi(t) d t \lesssim c_{N}(\epsilon|\xi|)^{-N}
$$

Set $N=(c+1)\left(s_{B}-h\right) \geq 1$, where an additional positive lower bound on $c$ will be chosen momentarily. It is easy to check that $(\epsilon|\xi|)^{-N}<|\xi|^{-\left(s_{B}-h\right)}$ if $|\xi|>(1 / \epsilon)^{1+1 / c}$, and so we bound (5.13) by $c_{N} \cdot(\epsilon|\xi|)^{-N} \lesssim c_{N}|\xi|^{-\left(s_{B}-h\right)}$. Hence, Proposition 5.2 holds with constants independent of $\epsilon$ for $|\xi|>(1 / \epsilon)^{1+1 / c}$.

If $|\xi|>(1 / \epsilon)^{1+1 / c}$, we bound (5.13) by $\epsilon^{s_{B}-h}$ and see that for this range of $|\xi|$,

$$
\epsilon^{s_{B}-h} \lesssim|\xi|^{-\left(s_{B}-h\right)+\left(s_{B}-h\right) /(c+1)} .
$$

Choosing $c$ so large that $\left(s_{B}-h\right) /(c+1)<\eta$, we conclude our proof of Proposition 5.2 .

\section{A Appendix}

A.1 Proof of (2.3). We prove (2.3) by contradiction, using (2.2). Set

$$
C=\left\{x: \lambda(x)>s_{A}+s_{B}-d\right\},
$$

and assume that the $d$-dimensional Lebesgue measure of $C$ is positive. This implies that there exists a real number $N>0$ such that

$$
C_{N}=\left\{x: \lambda(x)>s_{A}+s_{B}-d+2 / N\right\}
$$


also has positive $d$-dimensional Lebesgue measure. We restrict our attention to $x \in C_{N}$.

We begin by rewriting the set $C_{N}$. For $j \in \mathbb{N}$, define

$$
D_{N, J}=\left\{x \in C_{N}: \lambda(x)-\frac{1}{N}<\frac{\log (N(x, \epsilon))}{\log (1 / \epsilon)} \text { for all } 0<\epsilon \leq 2^{-j}\right\} .
$$

We claim that $C_{N}=\bigcup_{j=1}^{\infty} D_{N, j}$. Obviously, $\bigcup_{j=1}^{\infty} D_{N, j} \subset C_{N}$. On the other hand, for $x \in C_{N}, A \cap(s(x)-B) \neq \varnothing$ since $x \in C_{N}$ implies that $\lambda(x)>s_{A}+s_{B}-d+2 / N>$ 0 . Moreover, since $A \cap(s(x)-B) \neq \varnothing$,

$$
\lambda(x)=\liminf _{\epsilon \downarrow 0}\left(\frac{\log (N(x, \epsilon))}{\log (1 / \epsilon)}\right)=\lim _{\delta \downarrow 0}\left(\inf \left\{\frac{\log (N(x, \epsilon))}{\log (1 / \epsilon)}: 0<\epsilon \leq \delta\right\}\right) .
$$

By the definition of the limit, there exists $j \in \mathbb{N}$ such that

$$
\lambda(x)-\frac{1}{N}<\inf \left\{\frac{\log (N(x, \epsilon))}{\log (1 / \epsilon)}: 0<\epsilon \leq 2^{-j}\right\} .
$$

Hence, by the definition of the infimum,

$$
\lambda(x)-\frac{1}{N}<\frac{\log (N(x, \epsilon))}{\log (1 / \epsilon)}
$$

for all $0<\epsilon \leq 2^{-j}$, This shows that $x \in D_{N, j}$ and thus proves the claim.

Observe that $D_{N, j} \subset D_{N, j+1}$ and recall that $\mathcal{L}^{d}\left(C_{N}\right)>0$. Since $C_{N}=\bigcup_{j=1}^{\infty} D_{N, j}$, it follows that

$$
\mathcal{L}^{d}\left(\bigcup_{j=1}^{J} D_{N, j}\right)=\mathcal{L}^{d}\left(D_{N, J}\right)>0
$$

for some sufficiently large $J$.

To summarize, we have found a set $D_{N, J} \subset C_{N}$ of positive Lebesgue measure with $J$ sufficiently large such that $x \in D_{N, J}$ implies

$$
s_{A}+s_{B}-d+\frac{1}{N}<\lambda(x)-\frac{1}{N}<\frac{\log (N(x, \epsilon))}{\log (1 / \epsilon)}
$$

for all $\epsilon \in\left(0,2^{-J}\right]$. It follows that for $x \in D_{N, J}$ and $\epsilon \in\left(0,2^{-J}\right]$,

$$
\left(\frac{1}{\epsilon}\right)^{s_{A}+s_{B}-d+\frac{1}{N}}<N(x, \epsilon) .
$$

Let $\psi_{J}$ be a smooth, compactly supported, non-negative function satisfying $\int_{D_{N, J}} \psi_{J}(x) d x=1$. Such a function exists since $D_{N, J}$ has positive Lebesgue measure. We then obtain

$$
\int_{D_{N, J}}\left(\frac{1}{\epsilon}\right)^{\left(s_{a}+s_{b}-d+\frac{1}{N}\right)} \psi_{J}(x) d x<\int_{D_{N, J}} N(x, \epsilon) \psi_{J}(x) d x .
$$


for $\epsilon \in\left(0,2^{-J}\right]$. Since $\int_{D_{N, J}} \psi_{J}(x) d x=1$, it follows that

$$
\left(\frac{1}{\epsilon}\right)^{\left(s_{A}+s_{B}-d+\frac{1}{N}\right)}<\int_{D_{N, J}} N(x, \epsilon) \psi_{J}(x) d x .
$$

Using (2.2) to bound the right-hand side of this expression, we obtain

$$
\left(\frac{1}{\epsilon}\right)^{\left(s_{A}+s_{B}-d+\frac{1}{N}\right)}<\int_{D_{N, J}} N(x, \epsilon) \psi_{J}(x) d x<C^{\prime}\left(\frac{1}{\epsilon}\right)^{\left(s_{A}+s_{B}-d\right)}
$$

for $\epsilon \in\left(0,2^{-J}\right]$, where $C^{\prime}>0$ is independent of $\epsilon$. However, (A.7) cannot hold for sufficiently small $\epsilon>0$; hence we have arrived at a contradiction. Therefore, $\lambda(x) \leq s_{A}+s_{B}-d$ for almost every $x \in \mathbb{R}^{d}$ with respect to Lebesgue measure.

A.2 Proof of Lemma 3.1. To prove that $g(x)$ is upper semi-continous at a point $x_{0}$, we find $\delta>0$ such that $\left|x-x_{0}\right|<\delta$ implies that $g(x) \leq g\left(x_{0}\right)$; i.e., we fix $\epsilon>0$ and find a $\delta>0$ such that $\left|x-x_{0}\right|<\delta$ implies

$$
N(x, \epsilon) \leq N\left(x_{0}, \epsilon\right) .
$$

We consider the cases $N\left(x_{0}, \epsilon\right)=0$ and $N\left(x_{0}, \epsilon\right) \neq 0$ separately. In both cases, we utilize the following simple fact, whose proof we omit.

Proposition A.1. Let $U$ be a non-empty open subset of $\mathbb{R}^{d}$ and $X$ a nonempty compact subset of $U$. There exists $\delta>0$ such that

$$
X^{\delta} \subset U \text {. }
$$

We now turn to the proof of (A.8) in the case $N\left(x_{0}, \epsilon\right)=0$. Note that $N\left(x_{0}, \epsilon\right)=$ 0 if and only if $A \cap\left(s\left(x_{0}\right)+B\right)=\varnothing$. In this case, $s\left(x_{0}\right)+B \subset \mathbb{R}^{d} \backslash A$. Since $s\left(x_{0}\right)+B$ is compact and $\mathbb{R}^{d} \backslash A$ is open, it follows by Proposition A. 1 that there exists $\lambda>0$ such that $\left\{s\left(x_{0}\right)+B\right\}^{\lambda} \subset \mathbb{R}^{d} \backslash A$. By the continuity of $s$, there exists $\delta>0$ such that $\left|x-x_{0}\right|<\delta$ implies that $\left|s(x)-s\left(x_{0}\right)\right|<\lambda$, and so $s(x)+B \subset\left\{s\left(x_{0}\right)+B\right\}^{\lambda} \subset$ $\mathbb{R}^{d} \backslash A$. We have shown that there exists $\delta>0$ such that $\left|x-x_{0}\right|<\delta$ implies that $A \cap(s(x)+B)=\varnothing$, which establishes (A.8) for this case.

Now suppose $N\left(x_{0}, \epsilon\right) \neq 0$. For simplicity of notation, write $N=N\left(x_{0}, \epsilon\right)$. There exist $N$ open $\epsilon$-balls, $B_{\epsilon}\left(c_{1}\right), \ldots, B_{\epsilon}\left(c_{N}\right)$, such that

$$
A \cap\left(s\left(x_{0}\right)+B\right) \subset \bigcup_{i=1}^{N} B_{\epsilon}\left(c_{i}\right) .
$$

We claim that there exists $\delta>0$ such that $\left|x-x_{0}\right|<\delta$ implies

$$
A \cap(s(x)+B) \subset \bigcup_{i=1}^{N} B_{\epsilon}\left(c_{i}\right)
$$


This would complete the proof of (A.8), since (A.11) implies that $N(x, \epsilon) \leq N$. To find $\delta$ such that (A.11) holds, we first apply Proposition A.1 to (A.10) and conclude that there exists $\lambda>0$ such that

$$
\left\{A \cap\left(s\left(x_{0}\right)+B\right)\right\}^{\lambda} \subset \bigcup_{i=1}^{N} B_{\epsilon}\left(c_{i}\right) .
$$

We next claim that there exists $\delta>0$ such that $\left|x-x_{0}\right|<\delta$ implies

$$
A \cap(s(x)+B) \subset\left\{A \cap\left(s\left(x_{0}\right)+B\right)\right\}^{\lambda} .
$$

Note that (A.11) follows from (A.12) and (A.13). To prove (A.13), we require the following result.

Proposition A.2. Let $A$ and $B$ be nonempty compact subsets of $\mathbb{R}^{d}$, and suppose that $\lambda>0$ is such that (A.12) holds. There exists $\eta>0$ such that

$$
A \cap(x+B) \subset\{A \cap B\}^{\lambda}
$$

whenever $|x|<\eta$.

Replacing $B$ in the statement of Proposition A.2 with $s\left(x_{0}\right)+B$ gives $\eta>0$ such that $|y|<\eta$ implies that $A \cap\left(y+s\left(x_{0}\right)+B\right) \subset\left\{A \cap\left(s\left(x_{0}\right)+B\right)\right\}^{\lambda}$. By the continuity of $s$, there exists $\delta>0$ such that $\left|x-x_{0}\right|<\delta$ implies that $\left|s(x)-s\left(x_{0}\right)\right|<\eta$, i.e., $\left|x-x_{0}\right|<\delta$ implies that $s(x)=y+s\left(x_{0}\right)$ for some $|y|<\eta$, and so

$$
A \cap(s(x)+B) \subset\left\{A \cap\left(s\left(x_{0}\right)+B\right)\right\}^{\lambda} .
$$

This completes the proof of (A.13), given Propositions A.1 and A.2.

A.3 Proof of Proposition A.2. Fix $\lambda>0$, and cover $B \cap A$ with the balls $B(b, \lambda / 2)$. Cover $B \cap\left(\mathbb{R}^{d} \backslash A\right)$ with balls $B\left(b, \gamma_{b}\right)$, where $\gamma_{b}$ is chosen so that $B\left(b, 2 \gamma_{b}\right) \cap A=\varnothing$ (this is possible because $A$ is closed). From this cover of $B$, extract a finite subcover $\left\{B\left(b_{1}, \lambda / 2\right), \ldots B\left(b_{N}, \lambda / 2\right), B\left(\tilde{b}_{1}, \gamma_{1}\right), \ldots, B\left(\tilde{b}_{M}, \gamma_{M}\right)\right\}$, where $b_{i} \in B \cap A$ and $\tilde{b} \in B \cap\left(\mathbb{R}^{d} \backslash A\right)$. Let $\delta=\min \left\{\lambda / 2, \gamma_{1}, \ldots, \gamma_{M}\right\}$. Now $|x|<\delta$ guarantees that $A \cap(x+B) \subset\{A \cap B\}^{\lambda}$. Indeed, let $v=x+b \in A$, where $b \in B$ and $|x|<\delta$. Clearly, $b \notin B\left(\tilde{b_{j}}, \gamma_{j}\right)$ for $j=1, \cdots, M$ (since otherwise, $\left|\tilde{b_{j}}-v\right| \leq\left|\tilde{b_{j}}-b\right|+|b-v|<\gamma_{j}+\delta \leq 2 \gamma_{j}$ which contradicts $B\left(\tilde{b_{j}}, 2 \gamma_{j}\right) \cap A=\varnothing$.) Therefore, $b \in B\left(b_{i}, \lambda / 2\right)$ for some $i \in\{1, \cdots, N\}$, where $b_{i} \in A \cap B$. Now, $\left|v-b_{i}\right| \leq|x|+\lambda / 2<\lambda$, and so $v \in\{A \cap B\}^{\lambda}$. 


\section{REFERENCES}

[1] S. Eswarathasan, A. Iosevich, and K. Taylor, Fourier integral operators, fractal sets, and the regular value theorem, Adv. Math. 228 (2011), 2385-2402.

[2] K. J. Falconer, On the Hausdorff dimensions of distance sets Mathematika 32 (1985), 206-212.

[3] K. J. Falconer, The Geometry of Fractal Sets, Cambridge University Press, Cambridge, 1986.

[4] K. J. Falconer, Sets with large intersection properties, J. London Math. Soc. (2) 49 (1994), $267-$ 280.

[5] H. Federer, Geometric Measure Theory, Springer-Verlag, New York, 1969.

[6] A. Iosevich, H. Jorati, and I. Laba, Geometric incidence theorems via Fourier analysis, Trans. Amer. Math. Soc. 361 (2009), 6595-6611.

[7] P. Mattila, Hausdorff dimension and capacities of intersections of sets in n-space, Acta Math. 152 (1984), 77-105.

[8] P. Mattila, On the Hausdorff dimension and capacities of intersections, Mathematika 32 (1985), 213-217.

[9] P. Mattila Spherical averages of Fourier transforms of measures with finite energy: dimensions of intersections and distance sets, Mathematika 34 (1987), 207-228.

[10] P. Mattila, Geometry of Sets and Measures in Euclidean Spaces, Cambridge University Press, Cambridge, 1995.

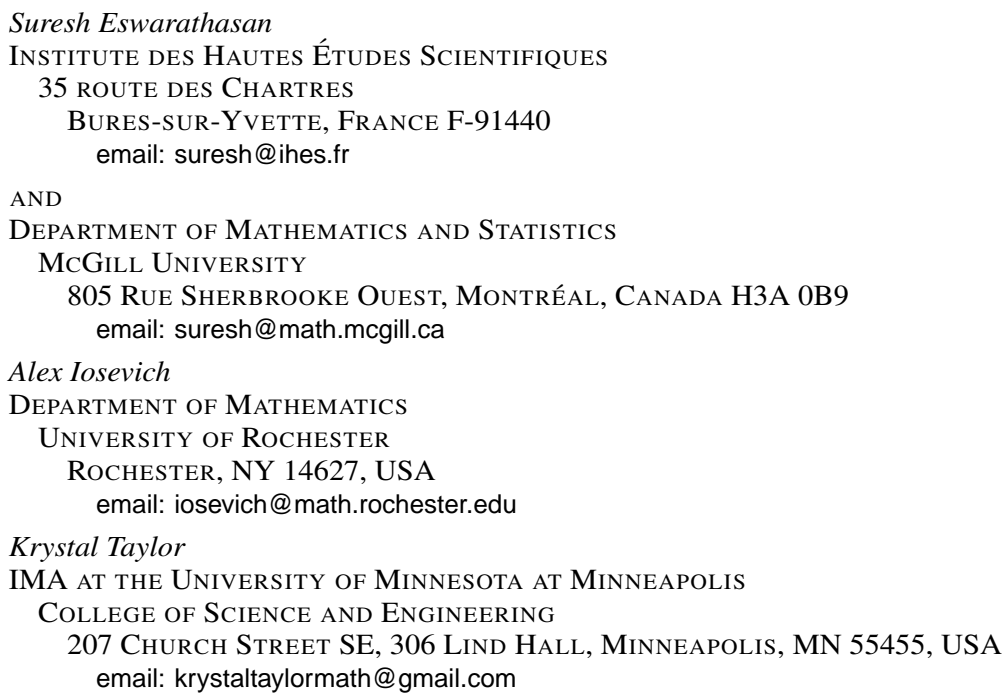

(Received and in revised form ) 